\title{
POTENCIAL CONTRIBUCIÓN DEL ARTE AL DESARROLLO DE HABILIDADES DE OBSERVACIÓN
}

\section{Potential contribution of art to the development of observation skills}

\begin{abstract}
Agustín HIDALGO BALSERA
Área de Farmacología. Universidad de Oviedo. Instituto Universitario de Oncología del Principado de Asturias (IUOPA). Instituto
\end{abstract} de Investigación Sanitaria de Asturias (ISPA) (España).

e-mail: hidalgo@uniovi.es

Las actividades formativas en humanidades médicas tienen el objetivo global de ayudar a que se desarrolle esa otra persona que está detrás del estudiante, a que se desarrolle como hombre y, de esta forma, se aproxime a la demanda de formar perfiles profesionales centrados en el paciente y no sólo en la enfermedad; es decir, de médicos portadores de valores humanos y conocedores de su aplicación. Por otra parte, la medicina es una profesión muy sensual ${ }^{1}$; es decir, requiere de los sentidos para su plena expresión. La observación y descripción de obras de arte puede contribuir tanto al desarrollo personal como profesional de los estudiantes y a la adquisición de competencias de observación a través del análisis de las experiencias de los enfermos y de los contextos social, cultural e histórico de la práctica médica en obras de arte, además de favorecer el desarrollo de la empatía, el altruismo o la compasión ${ }^{2}$.

La mayoría de los artículos publicados refieren que las actividades se realizan con estudiantes de cursos preclínicos, en grupos reducidos y, mayoritariamente, con la colaboración de instructores de arte de museos colaboradores. También la mayoría de las experiencias reconocen como objetivo principal mejorar el análisis visual mediante una observación estructurada con el fin de que sirva de entrenamiento para la observación clínica dado su carácter fundamental en la práctica asistencial. Nuestra experiencia se centra en la utilización de obras de arte para iniciar a los estudiantes en la adquisición de competencia de observación.

Para ello utilizamos dos seminarios dentro de una asignatura obligatoria denominada Introducción a la Medicina, Documentación y Método científico. En el primero (de entrenamiento), el profesor realiza una introducción general de los objetivos y comenta alguna imagen para que sirva de orientación a los estudiantes. Posteriormente, los estudiantes analizan una serie de obras y expresan sus comentarios sobre los que el profesor aporta las matizaciones que estime necesarias. En un segundo seminario, los estudiantes elaboran, mediante un análisis estructurado, una presentación sobre las obras que tienen asignadas e incorporan su trabajo al campus virtual para su evaluación.

En total hemos utilizado 90 obras pictóricas de 69 autores, creadas entre los siglos XV y XX, con predominio de los siglos XVII y XIX en las que los estudiantes han identificado 59 enfermedades o situaciones patológicas relacionadas con enfermedades infecciosas (con frecuencia en forma epidémica), afecciones del sistema 
nervioso central y adicciones, enfermedades endocrinas, del aparato digestivo y metabolismo, y enfermedades congénitas. Los enfermos generalmente aparecen representados en actitud yaciente, en una consulta, o en la sala de espera previa a ser recibidos por el médico. Con frecuencia se presenta a los enfermos acompañados por familiares o personal sanitario dedicado a los cuidados, pero en ocasiones están solos en la imagen, lo que sugiere la soledad de la enfermedad.

Así mismo, en los cuadros que hemos utilizado pueden ser identificados una serie de 45 actos médicos o actuaciones de profesionales sanitarios compatibles con las actuales, tales como acciones preventivas, la práctica de la anamnesis, la exploración diagnóstica, la hospitalización, la terapéutica médica, la práctica quirúrgica, la visita domiciliaria y la realización de cuidados a la cabecera del enfermo, además de acciones formativas de estudiantes de medicina o demostraciones anatómicas ${ }^{3}$.

La experiencia sugiere, por una parte, que las habilidades de documentación y comunicación están bien desarrolladas en los estudiantes que acceden al Grado en Medicina. También podemos considerar que, globalmente, han sido iniciados en competencias que pueden ser trasladadas a su ejercicio profesional, tales como el análisis visual estructurado, las habilidades clínicas de observación y comunicación y aspectos más humanísticos de la práctica médica tales como la empatía, el altruismo o la compasión que pueden matizar el biologicismo imperante en la medicina actual ${ }^{4}$.
Por último, más del $70 \%$ de los estudiantes está satisfecho con la actividad y más del $60 \%$ la considera relevante para su formación. En consecuencia, consideramos que la actividad tiene interés formativo y puede contribuir a la adquisición de las competencias de observación en el médico en formación.

\section{Referencias}

1. González-García, M, Cantabrana B, Hidalgo A. La más sensorial de las profesiones. El papel de los sentidos en la comunicación médica. Rev Med Cine [Internet] 2019; 15(2): 107-19.

2. Hidalgo A, Hidalgo C, Cantabrana B. Contribución del arte a la formación de los estudiantes de medicina. Rev Med Cine [Internet] 2018; 14(1): 53-62.

3. Hidalgo A, Bordallo J, Cantabrana B. Utilidad potencial de las artes visuales en la enseñanza de la medicina. Educ Med. 2018; 19(S3): 284-93.

4. Hidalgo A, Hidalgo C, Cantabrana B. Observación y descripción de obras de arte en la enseñanza de la medicina. Una experiencia en la Universidad de Oviedo. Rev Med Cine [Internet] 2018; 14(2): 115-22.

Resumen de la ponencia presentada en la Reunión científica La Revista de Medicina y Cine en los estudios de Ciencias de la Salud el día 7 de noviembre de 2019, en el marco del XXIV Congreso de la Sociedad Española de Educación Médica y V Congreso Hispano-Luso, celebrado en Salamanca del 6 al 8 de noviembre de 2019.

\begin{tabular}{|l|l|}
\hline Agustín Hidalgo Balsera es Licenciado y Doctor en Medicina por la Universidad Complutense \\
de Madrid y Profesor de Farmacología de la Universidad de Oviedo. Entre sus áreas de \\
interés se encuentra la repercusión social de los medicamentos y la representación social \\
de la medicina y la enfermedad a través de las manifestaciones artísticas y los medios de \\
divulgación científica y comunicación social.
\end{tabular}

